University of Nebraska - Lincoln

DigitalCommons@University of Nebraska - Lincoln

7-15-2003

\title{
Global geometry optimization of silicon clusters described by three empirical potentials
}

\author{
S. Yoo \\ University of Nebraska-Lincoln \\ Xiao Cheng Zeng \\ University of Nebraska-Lincoln, xzeng1@unl.edu
}

Follow this and additional works at: https://digitalcommons.unl.edu/chemzeng

Part of the Chemistry Commons

Yoo, S. and Zeng, Xiao Cheng, "Global geometry optimization of silicon clusters described by three empirical potentials" (2003). Xiao Cheng Zeng Publications. 33.

https://digitalcommons.unl.edu/chemzeng/33

This Article is brought to you for free and open access by the Published Research - Department of Chemistry at DigitalCommons@University of Nebraska - Lincoln. It has been accepted for inclusion in Xiao Cheng Zeng Publications by an authorized administrator of DigitalCommons@University of Nebraska - Lincoln. 


\title{
Global geometry optimization of silicon clusters described by three empirical potentials
}

\author{
S. Yoo and X. C. Zeng a) \\ Department of Chemistry, University of Nebraska-Lincoln, Lincoln, Nebraska 68588
}

(Received 12 March 2003; accepted 21 April 2003)

\begin{abstract}
The "basic-hopping" global optimization technique developed by Wales and Doye is employed to study the global minima of silicon clusters $\operatorname{Si}_{n}(3 \leqslant n \leqslant 30)$ with three empirical potentials: the Stillinger-Weber (SW), the modified Stillinger-Weber (MSW), and the Gong potentials. For the small-sized SW and Gong clusters $(3 \leqslant n \leqslant 15)$, it is found that the global minima obtained based on the basin-hopping method are identical to those reported by using the genetic algorithm [Iwamatsu, J. Chem. Phys. 112, 10976 (2000)], as well as with those by using molecular dynamics and the steepest-descent quench (SDQ) method [Feuston, Kalia, and Vashishta, Phys. Rev. B 37, 6297 (1988)]. However, for the mid-sized SW clusters $(16 \leqslant n \leqslant 20)$, the global minima obtained differ from those based on the SDQ method, e.g., the appearance of the endohedral atom with fivefold coordination starting at $n=17$, as opposed to $n=19$. For larger SW clusters $(20 \leqslant n$ $\leqslant 30$ ), it is found that the "bulklike" endohedral atom with tetrahedral coordination starts at $n$ $=20$. In particular, the overall structural features of $\mathrm{SW} \mathrm{Si}_{21}, \mathrm{Si}_{23}, \mathrm{Si}_{25}$, and $\mathrm{Si}_{28}$ are nearly identical to the MSW counterparts. With the $\mathrm{SW} \mathrm{Si}_{21}$ as the starting structure, a geometric optimization at the B3LYP/6-31G $(d)$ level of density-functional theory yields an isomer similar to the ground-stateisomer of $\mathrm{Si}_{21}$ reported by Pederson et al. [Phys. Rev. B 54, 2863 (1996)]. (C) 2003 American Institute of Physics. [DOI: 10.1063/1.1581849]
\end{abstract}

\section{INTRODUCTION}

Small silicon clusters are of both fundamental and technological importance. Over the past two decades or so, small silicon clusters have been extensively studied both experimentally ${ }^{1-8}$ and theoretically. ${ }^{9-29} \mathrm{~A}$ central issue concerning the small clusters $\mathrm{Si}_{n}$ is their lowest-energy geometric structures, namely, their global minima as a function of the cluster size $n$. For $n \leqslant 7$, the global minima are firmly established by both $a b$ initio calculations and Raman or infrared spectroscopy measurements, whereas for $n \leqslant 13$ the global minima are also well established by $a b$ initio calculations. ${ }^{10,28,29}$ For $14 \leqslant n \leqslant 20$, the global minima have been predicted based on semiempirical tight-binding (TB) and density functional theory (DFT) calculations ${ }^{23,24}$ coupled with the genetic algorithm (GA) global optimization technique. ${ }^{30}$ For $\mathrm{Si}_{20}$, in particular, the global minimum has been confirmed by the quantum Monte Carlo calculation. ${ }^{26}$ For $n \geqslant 21$, a priori (unbiased) search for the global minima from either $a b$ initio or semiempirical calculations is yet to be done. To our knowledge, only for $\mathrm{Si}_{21}$ and $\mathrm{Si}_{25}$, candidates for the global minima have been suggested based on $a b$ initio calculations. ${ }^{19,26}$

For $n \leqslant 20$ cationic silicon clusters, mobility experiments have revealed that a structural transition from a prolate to a "more spherical" geometry occurs in between $24 \leqslant n \leqslant 27 .{ }^{4}$ For neutral silicon clusters, however, photoionization experiments ${ }^{8}$ have shown that the prolate-to-spherical-like structural transition is likely in between $20 \leqslant n \leqslant 22$. On the theoretical side, an early $a b$ initio calculation suggested that

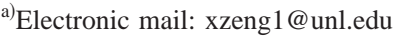

the critical size for the structural transition is bounded by $24 \leqslant n \leqslant 28 .{ }^{14}$ A more recent semiempirical TB and DFT study ${ }^{23}$ indicated that the transition may occur at $n=19$ because the spherical-like $\mathrm{Si}_{19}$ isomer with an endohedral atom becomes slightly more stable than the prolate isomer.

The global minima of silicon clusters have also been studied on the basis of the Stillinger-Weber (SW) and Gong empirical potentials. ${ }^{31-36}$ The SW potential was developed to reproduce a variety of bulk solid and liquid properties of silicon, ${ }^{35}$ and thus the global minima of SW clusters are not expected to be the same as the realistic global minima, especially for small-sized silicon clusters. Recently, Mousseau and co-workers suggested a slightly modified StillingerWeber (MSW) potential to simulate properties of amorphous silicon. ${ }^{31}$ Again, the global minima of MSW are not expected to be the same as those based on ab initio calculations. Gong also proposed a modified SW potential (hereafter called the Gong potential) in order to capture certain structural features of small-sized silicon clusters based on $a b$ initio calculations. ${ }^{36}$ Thus, it will be interesting to examine how well the Gong potential can describe the mid-sized clusters.

A number of methods have been developed for searching global minima. ${ }^{38}$ An early one is the simulated annealing (SA) method, which attempts to mimic real annealing experiments, namely, the target system is gradually cooled toward the zero temperature after being equilibrated at high temperatures. The SA method has been employed previously to search for the global minima of SW clusters $(3 \leqslant n \leqslant 17) .{ }^{31} \mathrm{It}$ is known that the SA method can be inefficient to locate the global minima of mid-size clusters since the system can be easily trapped in some metastable configurations when the 
temperature becomes too low. ${ }^{39}$ Recognizing the inefficiency of the SA method, Feuston et al. developed a new computational technique which combines the steepest-descent quench (SDQ) with the molecular dynamics simulation. ${ }^{32,33}$ Performing many SDQs in parallel allows one to determine all the statistically important potential-energy local minima in the configuration space and identify the lowest as the global minimum. Using the SDQ method, Feuston et al. found that the SA method ${ }^{31}$ failed to locate the global minima of $\mathrm{Si}_{6}$, $\mathrm{Si}_{11}$, and $\mathrm{Si}_{13}$ of the $\mathrm{SW}$ clusters for $n \leqslant 14$.

Using the SW and Gong potentials, Iwamatsu has calculated the global minima of silicon clusters for $3 \leqslant n \leqslant 15$ based on the GA global optimization method. ${ }^{30} \mathrm{He}$ found that the global-minimum structures of the SW clusters are identical to those obtained via the SDQ method. Note that the GA method is inspired from genetic evolution of real life. The GA optimization generally starts with a population of random structures. Then three operations, selection, crossover, and mutation are used to search for the global minima. An advantage of the GA method is that the search process itself is independent of the potential-energy surface. However, the amount of computer memory required for the GA calculation can increase very fast as the number of populations increases.

Recently, another global optimization technique, the "basin hopping" method, ${ }^{38,40,41}$ has been developed and applied to the Lennard-Jones (LJ) clusters ${ }^{42,43}$ up to $n=147$ and water clusters ${ }^{44}$ up to $n=20$. This technique has been proved to be robust since it can locate the global minima of $\mathrm{LJ}_{38}$ and $\mathrm{LJ}_{75}$, two very difficult cases because of their multifunnel-like potential energy surfaces. Here, we apply the basin-hopping technique to locate the global minima of silicon clusters for $3 \leqslant n \leqslant 30$. First, we will employ both the SW and Gong potentials to compare the calculated global minima with those (for $n \leqslant 15$ ) based on the GA method and those (for $n \leqslant 20$ ) based on the SDQ technique. We will then use the MSW potential to examine the effects of changing three-body part of potential function on the global-minimum structures. Next, for $15 \leqslant n \leqslant 20$, the global minima obtained via the basin-hopping method will be compared with the available $a b$ initio or TB calculations. We will monitor the first appearance of the endohedral atom in the cluster and discuss the prolate-to-spherical-like structural transition.

\section{EMPIRICAL POTENTIALS FOR SILICON}

The Stillinger-Weber potential function of silicon ${ }^{25}$ contains two-body and three-body terms:

$$
V=\sum_{i<j} v_{2}(i, j)+\sum_{i<j<k} v_{3}(i, j, k),
$$

where $V$ is the potential energy of the system, and $v_{2}$ is the two-body potential given by

$$
v_{2}(i, j)=A\left(B r_{i j}^{-p}-r_{i j}^{-q}\right) \exp \left[\left(r_{i j}-a\right)^{-1}\right] \Theta\left(a-r_{i j}\right),
$$

where $r_{i j}$ is the distance between the $i$ th and $j$ th atom. The three-body potential $v_{3}$ in Eq. (1) is given by

$$
v_{3}(i, j, k)=h\left(r_{j i}, r_{k i}\right)+h\left(r_{k j}, r_{i j}\right)+h\left(r_{i k}, r_{j k}\right),
$$

where

$$
\begin{aligned}
h\left(r_{j i}, r_{k i}\right)= & \lambda \exp \left[\frac{\gamma}{r_{j i}-a}+\frac{\gamma}{r_{k i}-a}\right] \\
& \times\left(\cos \theta_{j i k}+1 / 3\right)^{2} \Theta\left(a-r_{j i}\right) \Theta\left(a-r_{k i}\right) .
\end{aligned}
$$

In Eqs. (2) and (4), $\Theta(x)$ is the Heaviside step function; $\theta_{j i k}$ is the bond angle formed by three atom $j-i-k$. The seven adjustable parameters $A, B, a, p, q, \lambda$, and $\gamma$ appearing in Eqs. (2) and (4) are given by ${ }^{35}$

$$
\begin{aligned}
& A=7.049556277, \quad B=0.60222455884, \\
& p=4, \quad q=0, \quad a=1.80, \\
& A=21.0, \quad \gamma=1.20 .
\end{aligned}
$$

The energy is in units of $\epsilon(\epsilon=50 \mathrm{kcal} / \mathrm{mol}=2.16826 \mathrm{eV})$ and the length is in units of $\sigma(\sigma=2.0951 \AA)$.

Recently, Mousseau and co-workers ${ }^{37}$ proposed a MSW potential to better describe the tetrahedral structural characteristics of amorphous silicon. Specifically, one adjustable parameter is assigned to a new value, i.e., $\gamma=31.5$ and $\epsilon$ is reassigned as $1.64833 \mathrm{eV}$. This modification of the SW potential results in good agreement with the experimentally measured radial distribution function of the amorphous silicon. Because the $\lambda$ parameter in Eq. (4) characterizes a penalty to the three-body potential for the angular deviation from the tetrahedral coordination, the MSW potential is less tolerant, compared to the SW potential, to an angular deviation from the tetrahedral coordination.

Both SW and MSW potentials were developed to describe properties of bulk silicon. The Gong potential, ${ }^{36}$ on the other hand, was designed mainly to model small-sized silicon clusters. Gong noted from earlier ab initio calculations that small-sized silicon clusters exhibit a preferred bond angle of $\sim 60^{\circ}$. To reproduce this feature, Gong modified the three-body term $v_{3}$ with

$$
\begin{aligned}
h\left(r_{j i}, r_{k i}\right)= & \lambda \exp \left(\gamma\left(\left(r_{j i}-a\right)^{-1}+\left(r_{k i}-a\right)^{-1}\right)\right) \\
& \times\left(\cos \theta_{j i k}+1 / 3\right)^{2} \\
& \times\left[\left(\cos \theta_{j i k}+c_{0}\right)^{2}+c_{1}\right] \Theta\left(a-r_{j i}\right) \Theta\left(a-r_{k i}\right) .
\end{aligned}
$$

The two new adjustable parameters $c_{0}$ and $c_{1}$, and the $\lambda$ parameter are given by

$$
c_{0}=-0.5, \quad c_{1}=0.45, \quad \lambda=25.0 .
$$

TABLE I. The point group and potential energy per atom $(V / n)$ of the global minima of $\mathrm{Si}_{n}(n=3-15)$ based on the MSW potential. The energy is in units of $\epsilon$.

\begin{tabular}{cccccc}
\hline \hline Cluster & Point group & $V / n$ & Cluster & Point group & $V / n$ \\
\hline $\mathrm{Si}_{3}$ & $C_{2 v}$ & -0.6667 & $\mathrm{Si}_{10}$ & $D_{5 h}$ & -1.3181 \\
$\mathrm{Si}_{4}$ & $D_{4 h}$ & -0.9049 & $\mathrm{Si}_{11}$ & $C_{2 v}$ & -1.3397 \\
$\mathrm{Si}_{5}$ & $D_{5 h}$ & -0.9995 & $\mathrm{Si}_{12}$ & $D_{2 d}$ & -1.3745 \\
$\mathrm{Si}_{6}$ & $C_{2 v}$ & -1.0448 & $\mathrm{Si}_{13}$ & $C_{2 v}$ & -1.3883 \\
$\mathrm{Si}_{7}$ & $C_{3 v}$ & -1.1241 & $\mathrm{Si}_{14}$ & $D_{3 h}$ & -1.4154 \\
$\mathrm{Si}_{8}$ & $O_{h}$ & -1.2365 & $\mathrm{Si}_{15}$ & $C_{1}$ & -1.4084 \\
$\mathrm{Si}_{9}$ & $C_{2 v}$ & -1.2684 & & & \\
\hline \hline
\end{tabular}




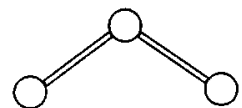

$\mathrm{n}=3, \mathrm{C}_{2 v}$

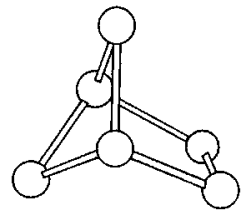

$\mathrm{n}=6, \mathrm{C}_{2 v}$

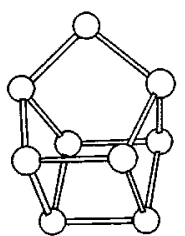

$\mathbf{n}=9, \mathbf{C}_{2 v}$

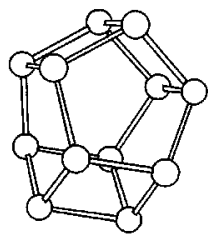

$\mathrm{n}=12, \mathrm{D}_{2 d}$

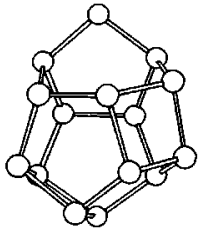

$\mathbf{n}=15, \mathbf{C}_{1}$

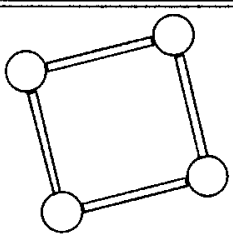

$\mathrm{n}=4, \mathrm{D}_{4 h}$

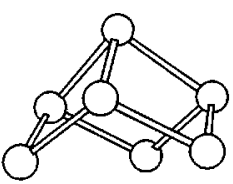

$\mathbf{n}=7, \mathrm{C}_{3 v}$

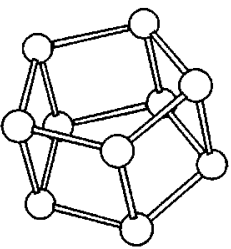

$\mathrm{n}=10, \mathrm{D}_{5 h}$

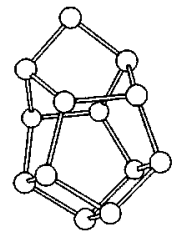

$\mathrm{n}=13, \mathrm{C}_{2 v}$
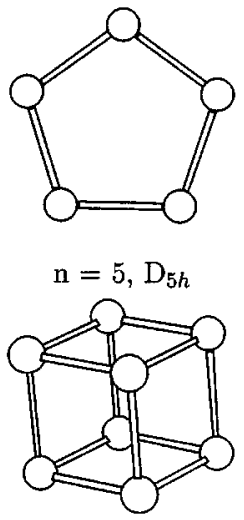

$\mathrm{n}=8, \mathrm{O}_{h}$
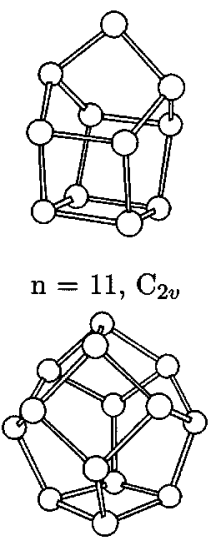

$\mathbf{n}=14, \mathrm{D}_{3 h}$

FIG. 1. Global minima of $\mathrm{Si}_{n}$ clusters $(n=3-15)$ based on the basin-hopping method and the MSW empirical potential.

Although the SW and MSW potentials are designed for the bulk phases whereas the Gong potential is designed for small-sized clusters, the three potentials all give the same value of the potential energy (in units of $\epsilon$ ) for the perfect diamond-structure crystal. This is because the three-body part of the potential energy becomes zero for the diamond crystal.

\section{BASIN-HOPPING GLOBAL OPTIMIZATION METHOD}

Here we give a brief summary of the "basin-hopping" global optimization technique. More details about this technique can be found elsewhere. ${ }^{40,41}$ Let $\mathbf{X}$ denote the vector of nuclear coordinates of a cluster. The ultimate outcome, by using the "basin-hopping" method, is a transformed potential energy surface $\widetilde{E}$ generated via the mapping

$$
\widetilde{E}(\mathbf{X})=\min \{E(\mathbf{X})\},
$$

where min denotes that the energy minimization is performed for the system configuration starting from $\mathbf{X}$. The topography of the transformed potential surface will resemble a multidimensional staircase, with each step corresponding to the basin of attraction surrounding a particular local minimum. The basin of attraction represents a set of geometries from which energy minimization always leads to the local minimum. With the transformed potential energy surface, the intra-potential-well vibration can be removed, thereby the system can "hop" directly between local minima at each step.

In practice, the transformed potential energy surface $\widetilde{E}$ can be explored via canonical Monte Carlo (MC) simulation. At each MC step all coordinates are randomly displaced with an adjustable step size to yield an acceptance ratio of 0.5. The energy change $\delta \widetilde{E}$ for hopping between two minima is accepted with the probability of $\exp \left(-\delta \widetilde{E} / k_{B} T\right)$, where $k_{B}$ is the Boltzmann constant and $T$ is the temperature. Here, for 


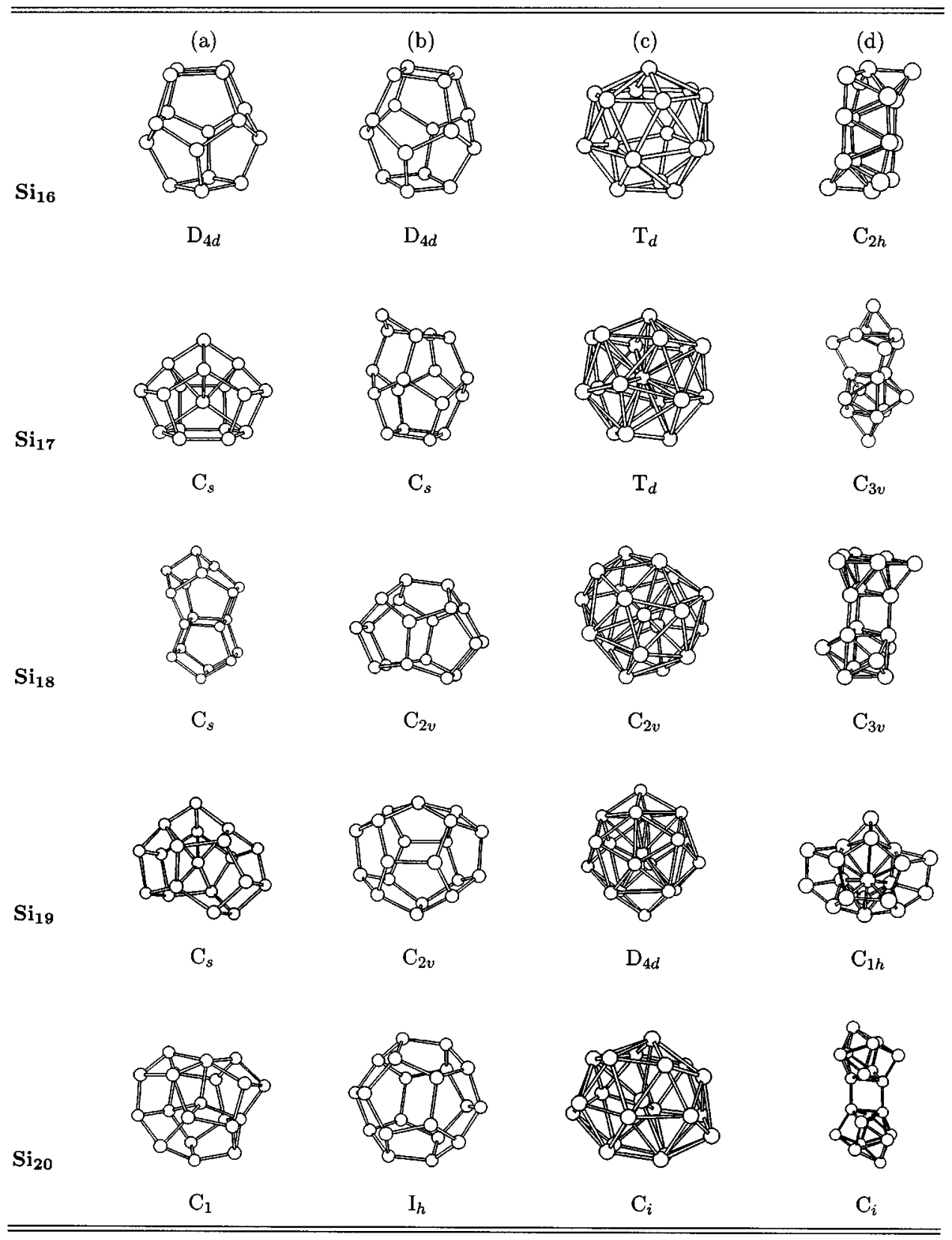

FIG. 2. Global minima of $\mathrm{Si}_{n}$ clusters $(n=16-20)$ based on the basin-hopping method and (a) the SW, (b) the MSW, and (c) the Gong empirical potentials. (d) Global minima based on the GA/TB/DFT calculation $(n=16-19$; Ref. 23) and quantum Monte Carlo simulation $(n=20$; Ref. 26).

each $\mathrm{Si}_{n}$ cluster $(3 \leqslant n \leqslant 30)$, five separate runs were carried out, each run consisting of 50000 energy quenches (minimizations) and each having a different starting configuration. The reduced temperature $T^{*}=k_{B} T / \epsilon=0.2$ was used. In most cases, the five runs yielded the same global minimum. For the MSW cluster $\mathrm{Si}_{28}$ and $\mathrm{Si}_{29}$, however, two runs resulted in an oblate local-minimum structure. When we set the temperature $T^{*}=0.25$, all five runs led to the same prolate global-minimum structure.

\section{RESULTS AND DISCUSSIONS}

\section{A. Small-sized clusters $(3 \leqslant n \leqslant 15)$}

Using the basin-hopping method we first calculate the global minima of the SW and Gong clusters $(3 \leqslant n \leqslant 15)$. The obtained potential energies per atom, the point groups, and the geometric structures are compared with the known results based on the $\mathrm{GA}^{34}$ and $\mathrm{SDQ}^{33}$ calculations. We find that our results reproduce exactly the known global minima of SW and Gong clusters, except for a few point-group assignments. For example, we find that the point group for $\mathrm{SW} \mathrm{Si}_{12}$ is $D_{2 d}$ and $\mathrm{SW} \mathrm{Si}_{14}$ is $D_{3 h}$. The point-group assignment for the Gong $\mathrm{Si}_{8}, \mathrm{Si}_{10}$ and $\mathrm{Si}_{15}$ is $D_{2 d}, D_{4 d}$, and $D_{3 h}$, respectively. Next, we calculate the global minima for small MSW clusters $(3 \leqslant n \leqslant 15)$. Their point groups and energies per atom are shown in Table I. The geometry of the global minima is plotted in Fig. 1, where bonds are defined as nearestneighbor distances less than $3 \AA$.

The overall global-minimum structures of MSW and SW clusters are nearly identical, except $\mathrm{Si}_{6}$ and $\mathrm{Si}_{13}$. This indi- 
(a)

$\mathbf{S i}_{21}$

$\mathrm{Si}_{22}$

$\mathrm{Si}_{23}$

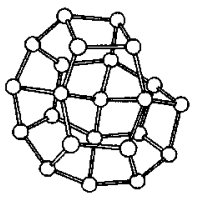

$\mathrm{C}_{2}$

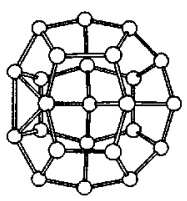

$\mathrm{C}_{s}$

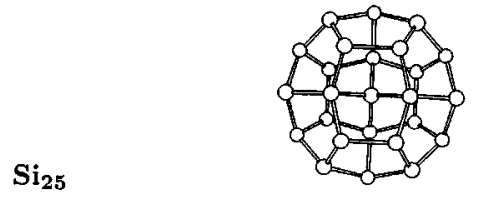

(b)

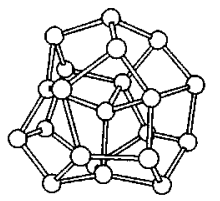

$\mathrm{T}$

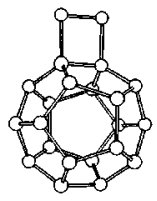

$\mathrm{C}_{2 v}$

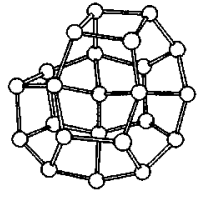

$\mathrm{C}_{2}$

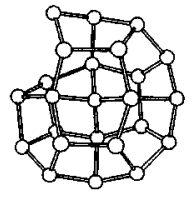

$\mathrm{C}_{1}$

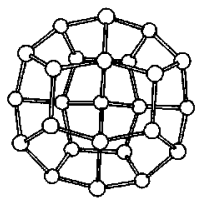

$\mathrm{D}_{2 d}$ (c)

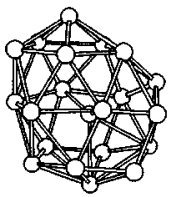

$\mathrm{C}_{2 v}$

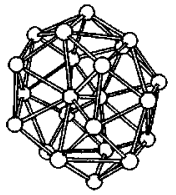

$\mathrm{D}_{2}$
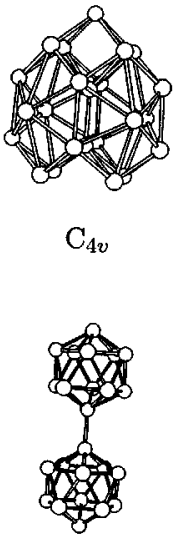

$\mathrm{D}_{5}$

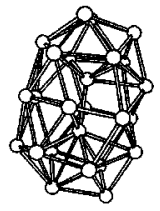

$\mathrm{C}_{s}$

FIG. 3. Global minima of $\mathrm{Si}_{n}$ clusters $(n=21-25)$ based on the basin-hopping method and (a) the SW, (b) the MSW, and (c) the Gong empirical potentials.

cates that changing the magnitude of the $\lambda$ parameter in the three-body potential has very little effects on the globalminimum structures of the small-sized SW clusters, in other words, the two-body potential appears to play the major role to give rise to the global-minimum structure for most smallsized clusters. As expected, none of the SW and MSW global minima is the same as the realistic global minima obtained from experiments or $a b$ initio calculations. Among the Gong clusters, however, the global-minimum structures of $\mathrm{Si}_{5}$ and $\mathrm{Si}_{7}$ are the same as those from the experiments ${ }^{6}$ as well as all electron molecular-orbital calculations. ${ }^{10,29}$

\section{B. Mid-sized clusters $(16 \leqslant n \leqslant 30)$}

Figures 2-4 display the global minima of mid-sized SW, MSW, and Gong clusters. Their potential energies per atom and the point groups are given in Table II. In Fig. 2(d), we also plotted the predicted global minima of silicon clusters based on GA/TB/DFT calculations (for $16 \leqslant n \leqslant 19)^{23}$ and $a b$ initio quantum Monte Carlo calculation (for $n=20$ ). ${ }^{26}$ First, the global minima of the mid-sized Gong clusters $(16 \leqslant n \leqslant 23)$ are all spherical-like and all the clusters exhibit a large number of $60^{\circ}$ bond angles. The latter result is due to the design of the Gong potential which is in favor of the bond angle of $60^{\circ}$ in addition to the tetrahedral bond angle. As a result, most silicon atoms in the Gong clusters have fivefold or sixfold coordination. The bond length is typically $2.55 \AA$, which is slightly longer than that of SW and MSW global minima $(2.35 \AA)$. As mentioned earlier, the Gong potential gives correct global-minimum geometry for smallsized cluster $\mathrm{Si}_{5}$ and $\mathrm{Si}_{7}$. For mid-sized clusters (e.g., $n$ $\geqslant 17$ ), the Gong clusters exhibit spherical-like structures which differ from the prolate structures predicted from the TB/DFT and $a b$ initio calculations. ${ }^{23,26}$ For $n \geqslant 17$, the Gong 


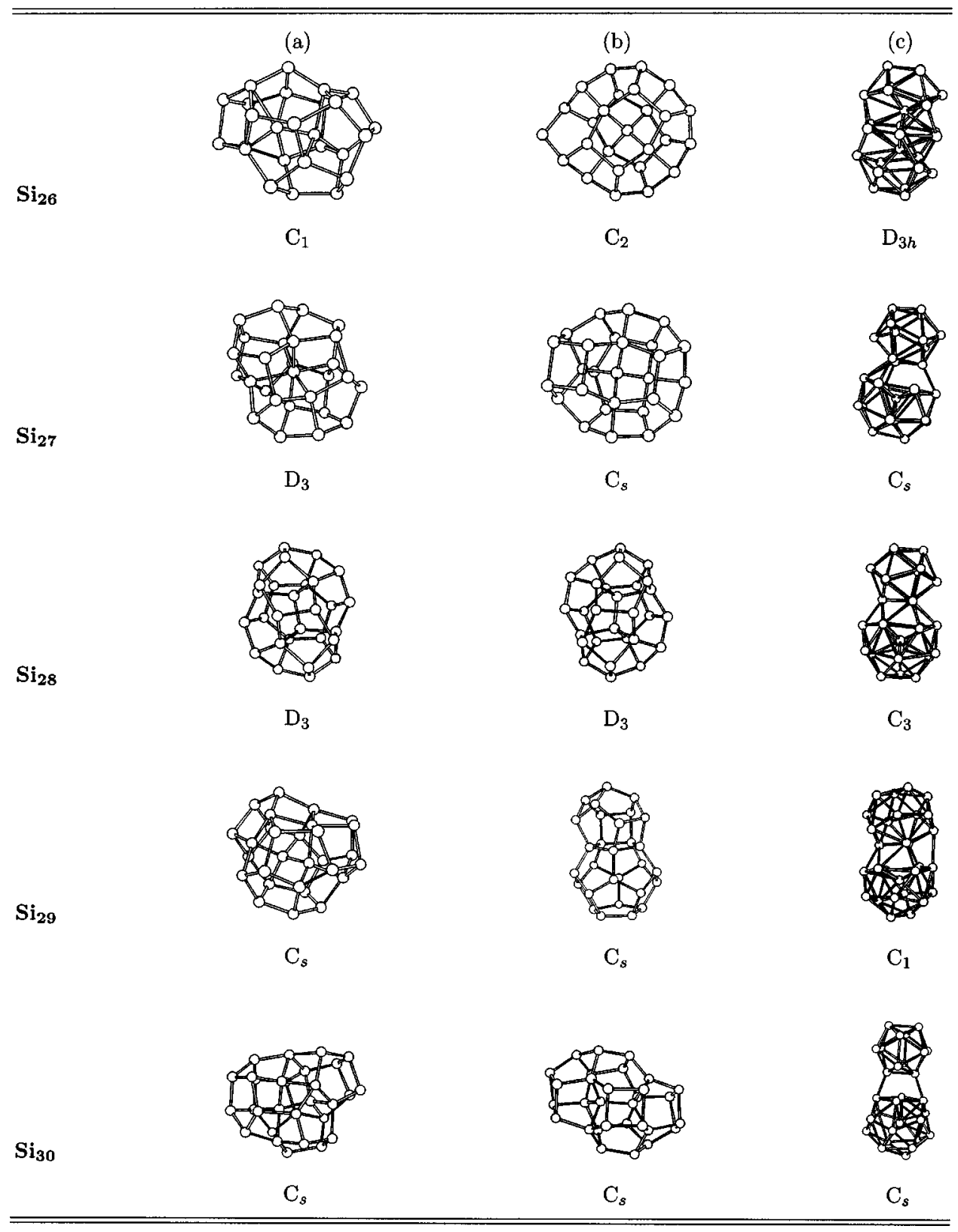

FIG. 4. The same as Fig. 3 but for $\mathrm{Si}_{n}$ clusters $(n=26-30)$.

clusters begin to show an endohedral atom with typically a very high coordination number. For $n \geqslant 24$, the Gong clusters show a spherical-to-prolate structural transition. This is in contrast to the mobility experiments and GA/TB calculations ${ }^{23}$ which show that the transition is from prolate to more-spherical-like as the size increases. We conclude that the Gong potential designed for small-sized silicon clusters appears to work well only for a few small-sized silicon clusters. Further improvements are needed in order to better describe other small-sized and mid-sized clusters.

Next, for mid-sized SW clusters $(16 \leqslant n \leqslant 20)$, it is found that the basin-hopping method results in different global minima compared to those based on the SDQ method. ${ }^{33}$ In particular, the appearance of the endohedral atom with fivefold coordination is found to start at $n=17$ as opposed to $n=19$. The energy calculations indicate that the global minima obtained based on the SDQ method were just very low-energy local minima. As mentioned in Sec. IV A, the SDQ method is essentially a method for scanning the potential-energy landscape from which one can locate statistically important local minima. In principle, given long enough molecular dynamics run time, the SDQ method should be able to locate the correct global minima, although this method can become inefficient for large-size clusters.

As in the case with the Gong potential, the SW potential also results in spherical-like global minima for most of the mid-sized clusters except for $n=18$. For $n \geqslant 20$, the SW clusters begin to show "bulklike" endohedral atoms with the tetrahedral coordination. This is due to the design of the SW potential which is to fit bulk properties of silicon. Similar behavior occurs for the MSW clusters except the "bulklike" endohedral atoms with the tetrahedral coordination beginning at $n=21$.

As mentioned in Sec. II, the SW potential differs from 
TABLE II. The point group and the potential energy per atom $(V / n)$ of the global minima of $\operatorname{Si}_{n}(n$ $=16-30$ ) based on the SW, MSW, and Gong potentials. The energy is in units of $\epsilon$.

\begin{tabular}{|c|c|c|c|c|c|c|}
\hline \multirow[b]{2}{*}{ Cluster } & \multicolumn{2}{|c|}{ SW } & \multicolumn{2}{|c|}{ MSW } & \multicolumn{2}{|c|}{ Gong } \\
\hline & Point group & $V / n$ & Point group & $V / n$ & Point group & $V / n$ \\
\hline $\mathrm{Si}_{16}$ & $D_{4 d}$ & -1.4677 & $D_{4 d}$ & -1.4499 & $T_{d}$ & -1.6456 \\
\hline $\mathrm{Si}_{17}$ & $C_{s}$ & -1.4724 & $C_{s}$ & -1.4413 & $T_{d}$ & -1.6850 \\
\hline $\mathrm{Si}_{18}$ & $C_{s}$ & -1.4788 & $C_{2 v}$ & -1.4506 & $C_{2 v}$ & -1.6947 \\
\hline $\mathrm{Si}_{19}$ & $C_{s}$ & -1.4995 & $C_{2 v}$ & -1.4529 & $D_{4 d}$ & -1.7060 \\
\hline $\mathrm{Si}_{20}$ & $C_{1}$ & -1.5094 & $I_{h}$ & -1.4984 & $C_{1}$ & -1.6801 \\
\hline $\mathrm{Si}_{21}$ & $T$ & -1.5372 & $T$ & -1.4852 & $C_{2 v}$ & -1.6807 \\
\hline $\mathrm{Si}_{22}$ & $C_{1}$ & -1.5209 & $C_{2 v}$ & -1.4777 & $D_{2}$ & -1.6757 \\
\hline $\mathrm{Si}_{23}$ & $C_{2}$ & -1.5368 & $C_{2}$ & -1.4965 & $C_{4 v}$ & -1.6787 \\
\hline $\mathrm{Si}_{24}$ & $C_{s}$ & -1.5371 & $C_{1}$ & -1.4959 & $D_{5}$ & -1.6979 \\
\hline $\mathrm{Si}_{25}$ & $D_{2 d}$ & -1.5512 & $D_{2 d}$ & -1.5246 & $C_{s}$ & -1.6986 \\
\hline $\mathrm{Si}_{26}$ & $C_{1}$ & -1.5519 & $C_{2}$ & -1.5154 & $D_{3 h}$ & -1.7181 \\
\hline $\mathrm{Si}_{27}$ & $D_{3}$ & -1.5598 & $C_{s}$ & -1.5216 & $C_{s}$ & -1.7138 \\
\hline $\mathrm{Si}_{28}$ & $D_{3}$ & -1.5720 & $D_{3}$ & -1.5232 & $C_{3}$ & -1.7136 \\
\hline $\mathrm{Si}_{29}$ & $C_{s}$ & -1.5636 & $C_{s}$ & -1.5330 & $C_{1}$ & -1.7106 \\
\hline $\mathrm{Si}_{30}$ & $C_{s}$ & -1.5707 & $C_{s}$ & -1.5352 & $C_{s}$ & -1.7191 \\
\hline
\end{tabular}

the MSW potential in the three-body term. The MSW gives greater penalty to the three-body potential for the angular deviation from the tetrahedral coordination than the SW. In Fig. 5, we plot the averaged bond-angle distributions for the 50 lowest-energy isomers of $\mathrm{SW}$ and $\mathrm{MSW} \mathrm{Si}_{26}$ and $\mathrm{Si}_{30}$. One can see that the MSW clusters show a much higher and sharper peak at the tetrahedral angles than the SW counterparts. Therefore, for the mid-sized clusters, even with the small difference in the three-body potential, it appears that the potential-energy landscape of the SW and MSW clusters can become quite different. Why are the global-minimum structures relatively insensitive to the minor change in the three-body potential when the cluster size is small whereas they become more sensitive to that change for the mid-sized clusters? One possible explanation is that for the mid-sized clusters there can exist many near-isoenergetic isomers. As a consequence, the global-minimum isomer of a SW cluster is likely to be a very low-energy isomer of the MSW counterpart. Thus, a small change in the three-body potential can still yield different global-minimum isomeric structures. It is interesting to observe that for clusters $\mathrm{Si}_{23}, \mathrm{Si}_{25}$, and $\mathrm{Si}_{28}$, SW and MSW potentials give nearly the same overall globalminimum structures. For $n=21$, the global-minimum structure of SW and MSW clusters is a centered dodecahedron with the tetrahedral point group $T$; the two structures appear to be mirror images. For $n=25$, the SW and MSW clusters are almost identical and both have an endohedral atom which is tetrahedrally bonded with four atoms on the outer shell. Moreover, the outer shell resembles the structure of the $\mathrm{Si}(111)$ surface. The quantum Monte Carlo simulation ${ }^{26}$ has shown that the ground-state structure of $\mathrm{Si}_{25}$ has three endohedral atoms, all with a higher coordination number (fivefold or sixfold). Finally, we note that the SW clusters $n$ $=22-24$ and the MSW clusters $n=23,24,26$, and 27 are all derivatives of the global minimum of $\mathrm{Si}_{25}$. For $n=28$, the global minimum exhibits two endohedral atoms and a surface reconstruction like that in $\mathrm{Si}_{21}$. Each endohedral atom is bonded with three atoms on the outer shell.

\section{C. $A b$ initio calculation of $\mathrm{SW} \mathbf{S i}_{21}$}

As mentioned in Sec. I, thus far, unbiased search for the global minima of silicon clusters from either ab initio or semiempirical calculations is limited to $n \leqslant 20$. For $n \geqslant 21$,

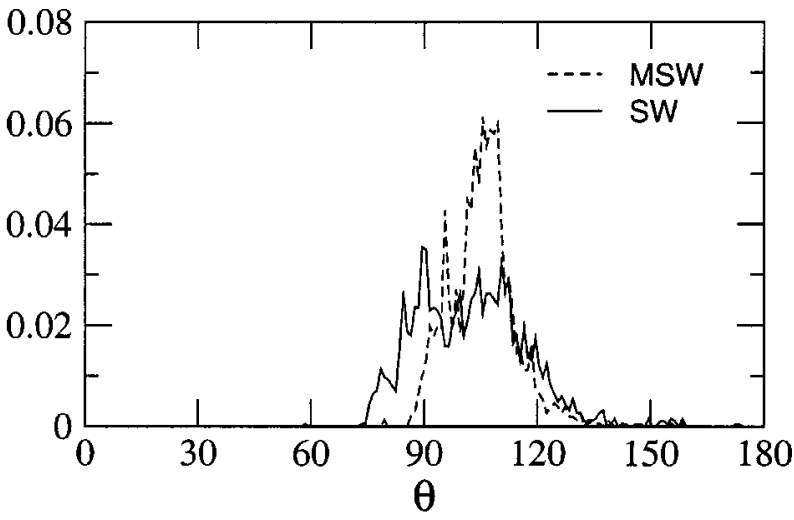

(a) $\mathrm{Si}_{26}$

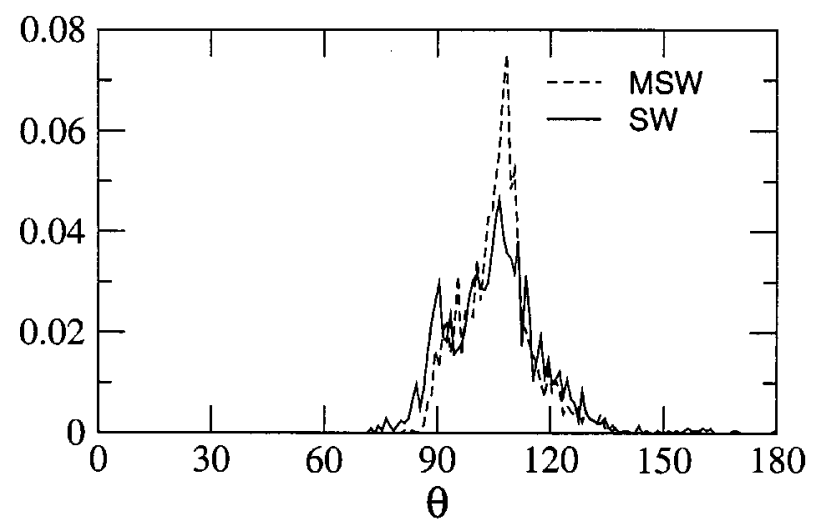

(b) $\mathrm{Si}_{30}$

FIG. 5. The averaged bond-angle distributions of the 50 lowest-energy isomers of (a) the SW and $\mathrm{MSW} \mathrm{Si}_{26}$ and (b) the $\mathrm{SW}$ and $\mathrm{MSW} \mathrm{Si}_{30}$. 


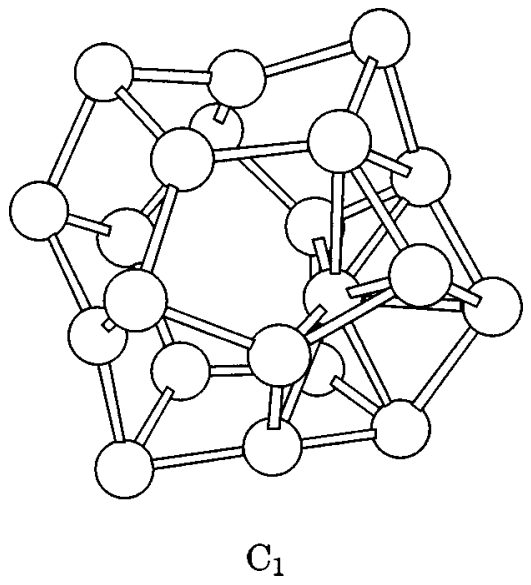

FIG. 6. The optimized structure of $\mathrm{Si}_{21}$ based on the ab initio DFT calculation at the B3LYP/6-31G(d) level. The starting structure is the $\mathrm{SW} \mathrm{Si}_{21}$ as shown in Fig. 3(a).

the search for the global minima via ab initio approaches has relied largely on physical insight into a specific cluster (see, for example, Refs. 13 and 14). By comparing the globalminimum isomers $(15 \leqslant n \leqslant 20)$ based on the SW and MSW potentials [Figs. 2(a) and 2(b)] with those based on TB/DFT and quantum Monte Carlo calculations [Fig. 2(d)], we suspect that if we use the global minima of SW or MSW isomers, which are spherical like, as the initial structures for $a b$ initio geometric relaxation, it is unlikely to yield the correct prolate-shaped global-minimum structures.

For $n \geqslant 21$, we notice that the overall global-minimum structures of $\mathrm{SW}$ and $\mathrm{MSW} \mathrm{Si}_{21}, \mathrm{Si}_{23}, \mathrm{Si}_{25}$, and $\mathrm{Si}_{28}$ are nearly identical (apart from a mirror symmetry) and that $\mathrm{SW}$ $\mathrm{Si}_{21}$ and $\mathrm{Si}_{28}$ as well as $\mathrm{MSW} \mathrm{Si}_{25}$ show relatively strong stability, compared to their nearest-neighbor clusters (Table II). Moreover, as mentioned in Sec. I, the prolate-tospherical-like structural transition is likely to occur at $n$ $=21$, and the SW clusters for $n \geqslant 21$ are indeed sphericallike. We therefore speculate that these SW isomer structures may serve as a good starting point for ab initio optimization to attain very low energy isomers, if not the global minima. To test this idea, we used the global minimum of $\mathrm{SW} \mathrm{Si}_{21}$ as the initial structure and performed an ab initio optimization at the B3LYP/6-31G $(d)$ level of DFT. ${ }^{45}$ Remarkably, we find that the optimized structure of $\mathrm{Si}_{21}$, we call it the DFT $\mathrm{Si}_{21}$ hereafter (see Fig. 6), is very similar to the ground-state structure predicted by Pederson and co-workers on the basis of the DFT calculation within the local-density approximation. ${ }^{19}$ The optimized structure of the DFT $\mathrm{Si}_{21}$ has a distorted cage composed of pentagonal rings. The endohedral silicon is relaxed toward the surface. We compared the bond-angle distribution of the DFT $\mathrm{Si}_{21}$ with that of the SW, MSW, and Gong clusters (Fig. 7). The global minima of SW and MSW exhibit similar bond-angle distribution with major peaks located at the tetrahedral angle and $120^{\circ}$. The Gong and DFT $\mathrm{Si}_{21}$ clusters, however, show additional peaks around $60^{\circ}$. (a) SW

(b) MSW
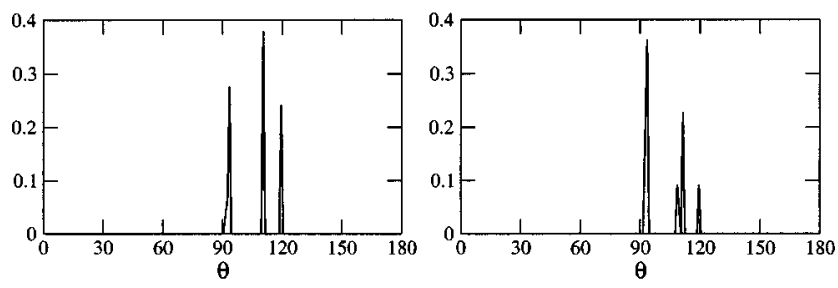

(c) Gong

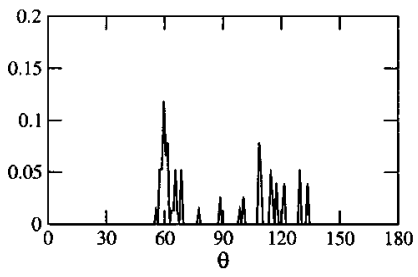

(d) DFT

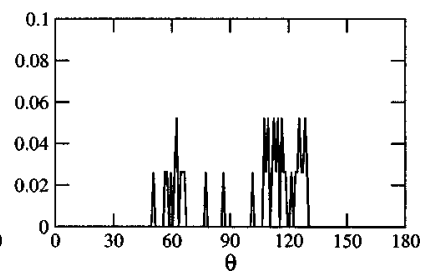

FIG. 7. The bond-angle distributions of the global minima of $\mathrm{Si}_{21}$ based on (a) the SW, (b) the MSW, and (c) the Gong potentials. (d) The bond-angle distribution of an isomer of $\mathrm{Si}_{21}$ based on the DFT geometry relaxation. The initial structure for the geometry relaxation is the $\mathrm{SW} \mathrm{Si}_{21}$.

\section{CONCLUSION}

The "basin-hopping" global optimization method has been applied to silicon clusters $3 \leqslant n \leqslant 30$. For $3 \leqslant n \leqslant 15$ the basin-hopping calculation reproduces the global minima of Gong and SW clusters based on the GA as well as the SDQ method. For the mid-sized clusters $(20 \leqslant n \leqslant 30)$ the geometries based on the SW and MSW potentials have the structural characteristics of the bulk silicon, such as four coordinated endohedral (internal) atoms. This is because the SW and MSW empirical potentials were designed for bulk silicon. In general, the global minima based on empirical potentials are not expected to be the same as those based on the $a b$ initio and semiempirical calculations. In fact, the $a b$ initio and TB calculations have shown that mid-sized clusters typically exhibit the structural characters of $\beta$-tin silicon. Finally, we note that because of their relatively strong stability as well as relative insensitivity to the change of the three-body potential, the global-minimum structures of $\mathrm{Si}_{21}, \mathrm{Si}_{25}$, and $\mathrm{Si}_{28}$ appear to be good starting structures for ab initio optimizations that may lead to very low energy or even lowestenergy isomer structures. Indeed, a preliminary ab initio calculation with using the $\mathrm{SW} \mathrm{Si}_{21}$ as the starting structure results in a possibly lowest-energy structure that has been reported previously based on the density-functional theory with the local-density approximation. Higher-level $a b$ initio calculations for these mid-sized silicon clusters are under way.

\section{ACKNOWLEDGMENTS}

We thank Dr. David Wales and Professor B. Pan and Professor X. L. Zhu for valuable discussions. This work is supported by the National Science Foundation and by the research computing facility at the University of NebraskaLincoln. 
${ }^{1}$ J. L. Elkind, J. M. Alford, F. D. Weiss, R. T. Laaksonene, and R. E. Smalley, J. Chem. Phys. 87, 2397 (1987).

${ }^{2}$ Q. L. Zhang, Y. Liu, R. F. Curl, F. K. Tittel, and R. E. Smalley, J. Chem. Phys. 88, 1670 (1988).

${ }^{3}$ M. F. Jarrold, Science 252, 1085 (1991).

${ }^{4}$ M. F. Jarrold and V. A. Constant, Phys. Rev. Lett. 67, 2994 (1991).

${ }^{5}$ M. F. Jarrold and J. E. Bower, J. Chem. Phys. 96, 9180 (1992).

${ }^{6}$ E. C. Honea, A. Ogura, C. A. Murray, K. Raghavachari, W. O. Sprenger, M. F. Jarrold, and W. L. Brown, Nature (London) 366, 42 (1993).

${ }^{7}$ S. Li, R. J. Van Zee, W. Weltner, Jr., and K. Raghavachari, Chem. Phys. Lett. 243, 275 (1995).

${ }^{8}$ K. Fuke, K. Tsukamoto, F. Misaizu, and M. Sanekata, J. Chem. Phys. 99, 7807 (1993).

${ }^{9}$ D. Tomanek and M. A. Schlüter, Phys. Rev. Lett. 56, 1055 (1986).

${ }^{10}$ K. Raghavachari and C. M. Rohlfing, J. Chem. Phys. 89, 2219 (1988).

${ }^{11}$ P. Ballone, W. Audreoni, R. Car, and M. Parrinello, Phys. Rev. Lett. 60, 271 (1988).

${ }^{12}$ B. C. Bolding and H. C. Andersen, Phys. Rev. B 41, 10568 (1990).

${ }^{13}$ E. Kaxiras, Phys. Rev. Lett. 64, 551 (1990).

${ }^{14}$ E. Kaxiras and K. Jackson, Phys. Rev. Lett. 71, 727 (1993).

${ }^{15}$ P. Ordejón, D. Lebedenko, and M. Menon, Phys. Rev. B 50, 5645 (1994).

${ }^{16}$ I. H. Lee, K. J. Chang, and Y. H. Lee, J. Phys.: Condens. Matter 6, 741 (1994).

${ }^{17}$ A. Bahel and M. V. Ramakrishna, Phys. Rev. B 51, 13849 (1995).

${ }^{18}$ J. C. Grossman and L. Mitáš, Phys. Rev. Lett. 95, 1323 (1995).

${ }^{19}$ M. R. Pederson, K. Jackson, D. V. Porezag, Z. Hajnal, and T. Frauenheim, Phys. Rev. B 54, 2863 (1996).

${ }^{20}$ A. Sieck, D. Porezag, Th. Frauenheim, M. R. Pederson, and K. Jackson, Phys. Rev. A 56, 4890 (1997).

${ }^{21}$ S. Wei, B. N. Barnett, and U. Landman, Phys. Rev. B 55, 7935 (1997).

${ }^{22}$ I. Vasiliev, S. Ogut, and J. R. Chelikowsky, Phys. Rev. Lett. 78, 4805 (1997).
${ }^{23}$ K.-M. Ho, A. A. Shvartsbug, B. Pan, Z.-Y. Lu, C.-Z. Wang, J. G. Wacker, J. L. Fye, and W. F. Jarrold, Nature (London) 392, 582 (1998).

${ }^{24}$ B. Liu, Z.-Y. Lu, B. Pan, C.-Z. Wang, K.-M. Ho, A. A. Shvartsbug, and M. F. Jarrold, J. Chem. Phys. 109, 9401 (1998).

${ }^{25}$ Y. Luo, J. Zhao, and G. H. Wang, Phys. Rev. B 60, 10703 (1999).

${ }^{26}$ L. Mitas, J. C. Grossman, I. Stich, and J. Tobik, Phys. Rev. Lett. 84, 1479 (2000).

${ }^{27}$ B. X. Li, P. L. Cao, and M. Jiang, Phys. Status Solidi 218, 399 (2000).

${ }^{28}$ Z.-Y. Lu, C.-Z. Wang, and K.-M. Ho, Phys. Rev. B 61, 2329 (2001).

${ }^{29}$ X. Zhu and X. C. Zeng, J. Chem. Phys. 118, 3558 (2003).

${ }^{30}$ J. H. Holland, Adaptation in Natural and Artificial Systems (University of Michigan Press, Ann Arbor, 1975).

${ }^{31}$ F. Blaisten-Barojas and D. Levesque, Phys. Rev. B 34, 3910 (1986).

${ }^{32}$ B. P. Feuston, R. K. Kalia, and P. Vashishta, Phys. Rev. B 35, 6222 (1987).

${ }^{33}$ B. P. Feuston, R. K. Kalia, and P. Vashishta, Phys. Rev. B 37, 6297 (1988).

${ }^{34}$ M. Iwamatsu, J. Chem. Phys. 112, 10976 (2000).

${ }^{35}$ F. H. Stillinger and T. A. Weber, Phys. Rev. B 31, 5262 (1985).

${ }^{36}$ X. G. Gong, Phys. Rev. B 47, 2329 (1993).

${ }^{37}$ R. L. C. Vink, G. T. Barkema, W. F. van der Weg, and N. Mousseau, J. Non-Cryst. Solids 282, 248 (2001).

${ }^{38}$ D. J. Wales and H. A. Scheraga, Science 285, 1368 (1999).

${ }^{39}$ R. Judson, Rev. Comput. Chem. 10, 1 (1997).

${ }^{40}$ D. J. Wales and J. P. K. Doye, J. Phys. Chem. A 101, 5111 (1997).

${ }^{41}$ D. J. Wales, M. A. Miller, and T. R. Walsh, Nature (London) 394, 758 (1998).

${ }^{42}$ J. P. K. Doye and D. J. Wales, Phys. Rev. Lett. 80, 1357 (1998).

${ }^{43}$ J. P. K. Doye, M. A. Miller, and D. J. Wales, J. Chem. Phys. 110, 6896 (1999).

${ }^{44}$ D. J. Wales and M. P. Hodges, Chem. Phys. Lett. 286, 65 (1998).

${ }^{45}$ M. J. Frisch et al., GAUSSIAN 98, Revision A.11, Gaussian, Inc., Pittsburgh, PA, 1998. 